\title{
Upfront metastasis-directed therapy in oligorecurrent prostate cancer does not decrease the time from initiation of androgen deprivation therapy to castration resistance
}

\author{
Luca Triggiani $^{1} \cdot$ Rosario Mazzola $^{2} \cdot$ Davide Tomasini $^{1}(1) \cdot$ Alessio Bruni $^{3} \cdot$ Giulia Alicino $^{3} \cdot$ Fabio Matrone $^{4}$. \\ Roberto Bortolus ${ }^{4}$. Giulio Francolini ${ }^{5}$. Beatrice Detti ${ }^{5}$ - Alessandro Magli ${ }^{6}$. Marco Lorenzo Bonù ${ }^{1}$. \\ Gianluca Ingrosso ${ }^{7}$. Andrea Lancia ${ }^{8} \cdot$ Fabio Trippa $^{9}$. Ernesto Maranzano ${ }^{9}$. Ciro Franzese ${ }^{10}$. Paolo Ghirardelli ${ }^{11}$. \\ Vittorio Vavassori ${ }^{11} \cdot$ Marta Scorsetti $^{10} \cdot$ Filippo Alongi $^{2}$. Stefano Maria Magrini ${ }^{1}$
}

Received: 23 March 2021 / Accepted: 29 April 2021 / Published online: 18 May 2021

(C) The Author(s) 2021

\begin{abstract}
The aim of the present study was to explore the potential impact of upfront metastases-directed therapy (MDT) in terms of prolongation of castration-sensitive phase in a series of oligorecurrent castration-sensitive prostate cancer (PC) patients. The present article is a multicenter retrospective study. The population of interest was castrate-sensitive oligorecurrent PC, defined as the presence of 1-3 uptakes in non-visceral sites such as bones or nodes detected by means of 18F-Choline PET/ CT or 68-Gallium PSMA PET/CT. Primary endpoint was the time to castration resistance. Secondary endpoints were ADTfree survival, local progression-free survival, and overall survival. Eighty-two patients and 118 lesions were analyzed. The median time to castration resistance for the entire population of the study was 49 months (95\% CI 43.6-54.4 months). The 1- and 2-year TTCR-free survival rates were $94 \%$ and $82 \%$, respectively. At the time of analysis, 52 patients were still in the castration-sensitive phase of the disease. In this cohort of patients, the median ADT-free survival was 20 months (range 3-69 months). On the other hand, during follow-up 30 patients switched to the castration-resistant phase of disease. In this last group of patients, the median ADT-free survival was 20 months (range 4-50 months). After the ADT administration, the median castration-sensitive phase was 29 months (range 5-71 months). Castration resistance generally occurs at a median follow-up of 24-36 months following ADT. In the current study, upfront MDT does not decrease the time from initiation of ADT to castration resistance.
\end{abstract}

Keywords Prostate cancer $\cdot$ Metastasis directed therapy $\cdot$ Stereotactic body radiotherapy

Davide Tomasini

tomad88@libero.it

1 Department of Radiation Oncology, University and Spedali Civili Hospital, Piazzale Spedali Civili 1, 25123 Brescia, Italy

2 Department of Advanced Radiation Oncology, IRCCS Sacro Cuore Don Calabria Hospital, Negrar Di Valpolicella, Verona, Italy

3 Department of Oncology and Hematology, Radiotherapy Unit, University Hospital of Modena, Modena, Italy

4 Department of Radiation Oncology, Centro Di Riferimento, Oncologico Di Aviano CRO-IRCCS, Aviano, Italy

5 Department of Radiation Oncology, University of Florence, A.O.U Careggi, Florence, Italy
6 Department of Radiation Oncology, University Hospital of Udine, ASUIUD, Udine, Italy

7 Radioterapia Oncologica, Dipartimento di Scienze Chirurgiche E Biomediche, Università degli Studi di Perugia, Ospedale S. Maria della Misericordia, Perugia, Italy

8 Radiation Oncology, Fondazione IRCCS Policlinico S. Matteo, Pavia, Italy

9 Department of Radiation Oncology, 'S. Maria' Hospital, Terni, Italy

10 IRCCS, Radiotherapy and Radiosurgery Department, Humanitas University Hospital, Milan-Rozzano, Italy

11 Cliniche Gavazzeni-Humanitas, Radiotherapy, Bergamo, Italy 


\section{Introduction}

Historically, the management of metastatic castrationsensitive prostate cancer (PC) relied on the administration of androgen deprivation therapy (ADT) with Luteinizing Hormone-Releasing Hormone (LH-RH) analogues or antagonist. In this clinical setting, ADT is able to prolong the biochemical control being able to impact on survival outcomes [1]. Unfortunately, ADT long-term effectiveness is limited, even if initial response with a fast PSA decrease is quite common after the first LHRH analogues administration. Indeed, after first-line ADT, disease progression represents a common event due to the development of the castration--resistant phase that generally occurs at a median follow-up of 24-36 months [2-4]. For this reason, ADT for metastatic PC is largely considered a palliative treatment and, in addition, the administration of ADT itself can be burdened by the onset of non-negligible adverse events such as cardiovascular events, metabolic syndrome, sarcopenia, sexual dysfunction, and osteoporosis [5]. These issues have to be particularly taken into account due to the long-term duration of this first line option and the relevance it may have particularly in frail or older patients. In the setting of oligorecurrent castration-sensitive PC a substantial amount of literature experiences, characterized by several levels of evidence, recently showed that metastasis-directed therapy (MDT) using high dose radiotherapy (SBRT) might represent a viable curative option able to improve disease control and significantly delay the administration of palliative ADT [6-8]. It is currently object of debate if MDT is able to prolong the time to castration resistance (TTCR) onset or negatively modify the natural history of the castration-sensitive PC.

The main aim of the present study was to explore the potential impact of upfront MDT in terms of prolongation of castration-sensitive phase in a series of oligorecurrent castration-sensitive PC patients.

\section{Methods}

The present article is a multicenter retrospective study. The inclusion criteria were (i) histologically confirmed diagnosis of acinar adenocarcinoma of the prostate, (ii) biochemical relapse after primary tumor treatment (radical prostatectomy or radical RT) defined according to European Association of Urology guidelines and/or according to Phoenix criteria in post-surgical or post-RT setting respectively [1], (iii) castrate-sensitive oligorecurrent PC, defined as the presence of 1-3 uptakes in non-visceral sites such as bones or nodes detected by means of
18F-Choline PET/CT (Cho-PET) or 68-Gallium PSMA PET/CT (PSMA PET), (iv) controlled primary tumor, (v) patients treated with upfront MDT for oligorecurrences without ADT, (vi) SBRT directed to every site of disease and delivered with $\geq 5$ Gy dose per fraction.

\section{SBRT and follow-up}

Before SBRT, all patients underwent a CT-based treatment planning with $1-3 \mathrm{~mm}$ slice thickness in the supine position. Gross tumor volume (GTV), equal to clinical target volume (CTV), was delineated using all the available morphological and metabolic imaging information. A planning target volume (PTV) was created around the CTV using isotropic or anisotropic 3-5 mm margins. Organs at risk were then delineated depending on the location of the target volume.

SBRT schedules followed the local treatment policy of each center varying between 25 and 50 Gy in 3 to 7 fractions. Image-guided intensity-modulated RT with static beams or dynamic arcs were used, depending on each center internal protocols and technology availability. At each fraction, the patient's set-up and target accuracy was always verified using on-board cone-beam or megavolt CT.

After SBRT, all patients were followed every 3-4 months with clinical evaluation and PSA blood tests. Cho-PET or PSMA PET scans were performed within 3 months after SBRT and every 3 months thereafter in the case of detectable PSA or biochemical progression post-SBRT, defined according to PC Working Group 3 [9]. Instrumental tumor response was assessed according to the PET Response Criteria in Solid Tumors [10].

If oligo-progression occurred after MDT, further courses of SBRT were proposed if less than 3 new lesions were detected by molecular imaging outside the previously irradiated field. Finally, ADT was administered when the conversion in the polymetastatic castration-sensitive phase of the disease occurred (more than three new metastases onset).

\section{Study endpoints and statistical analysis}

The primary endpoint of the current study was the TTCR defined according to EAU guidelines [1].

Secondary endpoints were ADT-free survival, local progression-free survival (LPFS), overall survival (OS). Detailed SBRT-related adverse events were also reported. TTCR was defined as the time between the starting of ADT after SBRT and three consecutive rising in PSA resulting in two $50 \%$ increases over the nadir and PSA $>2 \mathrm{ng} / \mathrm{ml}$ (castrate serum testosterone $<50 \mathrm{ng} / \mathrm{dl}$ ).

ADT-free survival was defined as the time between the last session of SBRT and the administration of ADT for the polymetastatic disease conversion. The LPFS was defined as the time between the last session of SBRT and 
the progression of the disease within the target volume. OS was calculated from the end of SBRT until the last follow-up or the patient's death.

Moreover, disease-free survival (DFS) was defined as the time between the primary treatment (surgery/radiotherapy) for prostate cancer at diagnosis and the first session of SBRT for oligorecurrent patients. Treatment-related toxicity was assessed using the Common Terminology Criteria for Adverse Events 5.0 (CTCAE 5.0) scale [11].

Descriptive statistics were used to summarize patient's features. Normality of the distributions was assessed using the Kolmogorov-Smirnov test. Categorical variables were presented as frequencies or percentages and compared with the use of the Chi-Square test or the Fisher's exact test, as appropriate. Continuous variables were presented as means \pm SD (in case of a normal distribution), or medians, IQR and $\min / \max$ (in case of a skewed distribution) and compared with the use of Student's t test, Anova, or the Mann-Whitney and Kruskal-Wallis test; correlations among variables by the Pearson's or Spearman's rank correlation test.

Logistic regression analyses were performed with the logrank test and the Kaplan-Meier method to correlate survival with the following variables: 1 or $>1$ metastases, sites of metastases (nodes and bone), location of node metastasis (N1 and M1a), primary tumor Gleason Score, D'Amico risk group, PSA values at the onset of metastases (dichotomized at the median value), and PSA doubling time (dichotomized at the median value). A $P$ value $<0.05$ was considered statistically significant. All statistical calculations were performed using SPSS Software version 20.0 (SPSS Inc, Chicago, IL).

\section{Results}

According to the inclusion criteria of the study, 82 patients and 118 lesions were retrospectively analyzed. In Table 1 baseline patients' and tumor characteristics are reported.

At the time of analysis, the median follow-up was 52 months (range 13-127 months). The DFS between primary tumor treatment and the onset of oligorecurrences was 55 months (range 33-92 months). Most patients (69/82) were affected by nodal oligometastases $(84.1 \%)$, while the remaining $13(15.9 \%)$ by bone metastases. Forty-two percent of patients were treated for single metastases, while the remaining 58\% received the SBRT treatment for two or more metastases. No patient was treated for more than three metastases.

The median TTCR for the entire population of the study was 49 months (95\% CI 43.6-54.4 months). The 1- and 2-year TTCR free survival rates were $94 \%$ and $82 \%$, respectively. Figure 1 depicts TTCR-free survival curve.
Table 1 Baseline patients and tumor characteristics

\begin{tabular}{|c|c|}
\hline Age at PCa diagnosis (yr), median (IQR) & $68(59-72)$ \\
\hline PSA at PCa diagnosis (ng/ml), median (IQR) & $10(7-15)$ \\
\hline \multicolumn{2}{|l|}{ c/pT Stage at diagnosis, n (\%) } \\
\hline $\mathrm{T} 1 \mathrm{c}$ & $1(1.2 \%)$ \\
\hline $\mathrm{T} 2$ & $33(40.2 \%)$ \\
\hline $\mathrm{T} 3$ & $43(52.4 \%)$ \\
\hline $\mathrm{T} 4$ & $5(6.1 \%)$ \\
\hline \multicolumn{2}{|l|}{$\mathrm{c} / \mathrm{p}$ N Stage at diagnosis, $\mathrm{n}(\%)$} \\
\hline No & $76(92.7 \%)$ \\
\hline N1 & $6(7.3 \%)$ \\
\hline \multicolumn{2}{|l|}{ Gleason Score sum at diagnosis, $\mathrm{n}(\%)$} \\
\hline 6 & $12(14.6 \%)$ \\
\hline 7 & $42(51.2 \%)$ \\
\hline$>7$ & $28(34.1 \%)$ \\
\hline \multicolumn{2}{|l|}{ D’Amico risk group, n (\%) } \\
\hline Very low/low & $8(9.7 \%)$ \\
\hline Intermediate (favorable/unfavorable) & $20(24.4 \%)$ \\
\hline High/very high & $54(65.9 \%)$ \\
\hline \multicolumn{2}{|l|}{ Type of primary treatment, $\mathrm{n}(\%)$} \\
\hline RP only & $15(18.3 \%)$ \\
\hline RT only & $13(15.9 \%)$ \\
\hline RP plus RT & $54(65.8 \%)$ \\
\hline \multicolumn{2}{|l|}{ RT field, n (\%) } \\
\hline Prostate only \pm seminal vesicles & $59(88 \%)$ \\
\hline Whole pelvis RT & $8(12 \%)$ \\
\hline \multicolumn{2}{|l|}{ ADT at primary treatment, $\mathrm{n}(\%)$} \\
\hline Yes & $29(35.4 \%)$ \\
\hline No & $53(64.6 \%)$ \\
\hline $\begin{array}{l}\text { Interval from diagnosis to oligometastases (mo), } \\
\text { median (IQR) }\end{array}$ & $55(33-92)$ \\
\hline Age at recurrence (yr) median (IQR) & $72(65-76)$ \\
\hline PSA at recurrence (ng/ml), median (IQR) & $1.3(0.6-2.3)$ \\
\hline \multicolumn{2}{|l|}{ Metastatic site, n (\%) } \\
\hline Nodal & $69(84.1 \%)$ \\
\hline Bone & $13(15.9 \%)$ \\
\hline \multicolumn{2}{|l|}{ Metastatic nodal site, $\mathrm{n}(\%)$} \\
\hline N1 & $47(68.1 \%)$ \\
\hline M1a & $17(24.6 \%)$ \\
\hline $\mathrm{N} 1+\mathrm{M} 1 \mathrm{a}$ & $5(7.2 \%)$ \\
\hline \multicolumn{2}{|l|}{ No. of lesions treated, n (\%) } \\
\hline 1 & $29(42 \%)$ \\
\hline$>2$ & $53(68 \%)$ \\
\hline \multicolumn{2}{|l|}{ SBRT schedule (node) } \\
\hline $35 \mathrm{~Gy} / 7 \mathrm{fr}$ & 19 \\
\hline $30 \mathrm{~Gy} / 5 \mathrm{fr}$ & 3 \\
\hline $36 \mathrm{~Gy} / 6 \mathrm{fr}$ & 17 \\
\hline $42 \mathrm{~Gy} / 7 \mathrm{fr}$ & 1 \\
\hline $31 \mathrm{~Gy} / 5 \mathrm{fr}$ & 1 \\
\hline $32 \mathrm{~Gy} / 5 \mathrm{fr}$ & 1 \\
\hline $35 \mathrm{~Gy} / 5 \mathrm{fr}$ & 3 \\
\hline $45 \mathrm{~Gy} / 6 \mathrm{fr}$ & 3 \\
\hline
\end{tabular}


Table 1 (continued)

\begin{tabular}{ll}
\hline $40 \mathrm{~Gy} / 5 \mathrm{fr}$ & 9 \\
$27 \mathrm{~Gy} / 3 \mathrm{fr}$ & 2 \\
$30 \mathrm{~Gy} / 3 \mathrm{fr}$ & 3 \\
$50 \mathrm{~Gy} / 5 \mathrm{fr}$ & 1 \\
$42 \mathrm{~Gy} / 4 \mathrm{fr}$ & 1 \\
$36 \mathrm{~Gy} / 3 \mathrm{fr}$ & 3 \\
$48 \mathrm{~Gy} / 4 \mathrm{fr}$ & 5 \\
$\mathrm{SBRT} \mathrm{schedule}($ Bone) & \\
$30 \mathrm{~Gy} / 3 \mathrm{fr}$ & 6 \\
$40 \mathrm{~Gy} / 5 \mathrm{fr}$ & 1 \\
$24 \mathrm{~Gy} / 3 \mathrm{fr}$ & 1 \\
$35 \mathrm{~Gy} / 5 \mathrm{fr}$ & 1 \\
$30 \mathrm{~Gy} / 5 \mathrm{fr}$ & 3 \\
$25 \mathrm{~Gy} / 5 \mathrm{fr}$ & 1 \\
\hline
\end{tabular}

The median ADT-free survival for the entire population of study was 18.2 months (95\% CI 14.5-21.9 months). The 1- and 2-year ADT-free survival rates were $66 \%$ and $32 \%$, respectively. At the time of analysis, 52 patients were still in the castration-sensitive phase of the disease. In this cohort of patients, the median ADT-free survival was 20 months (range 3-69 months). On the other hand, during follow-up 30 patients switched to the castration-resistant phase of disease. In this last group of patients, the median ADT-free survival was 20 months (range 4-50 months). After the ADT administration, the median castration-sensitive phase was 29 months (range 5-71 months).
Globally, 10-year OS was 96.3\%, whereas 5-year LPFS was $90.2 \%$. As far as adverse events are concerned, SBRT was well tolerated, with no gastrointestinal or genitourinary grade $\geq 2$ acute/late toxicity.

At statistical analyses, no correlation was found between the variables analyzed and the survival outcomes were analyzed, including the TTCR and ADTFS (Tables 2 and 3).

\section{Discussion}

The optimal combination of MDT and ADT is still a matter of study. During the last years, several studies have explored whether there could be a significant clinical impact by treating metastatic foci of disease by means of MDT in the scenario of oligorecurrent PC $[8,12]$.

It is true that most of the data are focused on the castration-sensitive oligorecurrent PC [13, 14]. Transversely, all the available literature data confirm the crucial role of MDT in terms of postponing drug administration as well as ameliorating certain survival endpoints [15]. Concerning the first point, the ADT administration delay is particularly important for clinicians to temporarily avoid ADT-related adverse events [16, 17]. Furthermore, in the recent years, ADT-free survival was also proposed as a surrogate end point of the other major clinical outcomes such as OS or PFS considering the importance of delaying an active but time-defined systemic treatment $[7,8]$.

On the other hand, it remains field of interest which patients could be the optimal therapeutic sequence: upfront MDT or first-line ADT followed by MDT or both in a

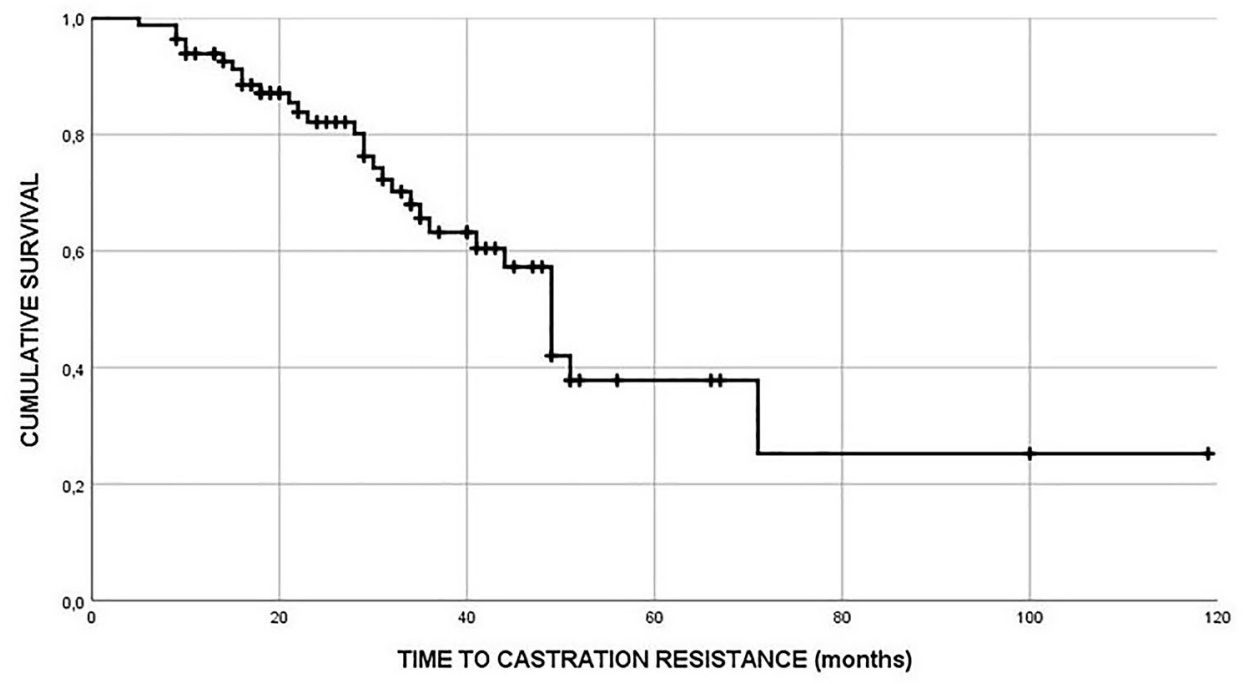

PATIENTS AT RISK

\begin{tabular}{|l|l|l|l|}
\hline 82 & 54 & 24 & 4 \\
\hline
\end{tabular}

4

2

1

1

Fig. 1 TTCR-free survival curve for the entire population (median 49 months, 95\% CI 43.6-54.4 months) 
Table 2 Statistical analytical results

\begin{tabular}{lll}
\hline Covariate & TTCR HR (95\% CI) & p-value \\
\hline D'Amico risk group, n (\%) & & \\
Very low/low & 1 & \\
Intermediate & $0.58(0.07-5.17)$ & 0.87 \\
High/very high & $0.75(0.09-5.71)$ & \\
Gleason score sum: & & \\
6 & 1 & \\
7 & $3.36(0.49-23.12)$ & \\
$>7$ & $5.24(0.67-41)$ & 0.27 \\
PSA level at time of metastases, ng/ & & \\
ml: & & \\
$<2$ ng/ml & 1 & \\
$\geq 2$ ng/ml & $0.68(0.28-1.64)$ & \\
Location of metastasis: & & \\
Node & 1 & 0.39 \\
Bone & $0.89(0.31-2.56)$ & \\
Location of node metastasis & & \\
N1 & 1 & \\
M1a & $1.41(0.53-3.73)$ & \\
No. of lesions & & \\
1 & $0.75(0.31-1.80)$ & \\
$>1$ & & \\
\hline
\end{tabular}

concomitant approach. During the St Gallen Advanced Prostate Cancer Consensus Conference [18], a disagreement was registered concerning the palliative ADT administration in the PC oligorecurrent patients. "ADT-believers" would administer first-line ADT to ensure its favorable effect on the natural history of castration-sensitive PC, whereas "MDT-believers" mostly appreciate the role of local ablative therapies (surgery or SBRT). Furthermore, a recent meta-analysis driven by Connor et al. comparing four randomized clinical trials showed that men with oligorecurrent castrate-sensitive PC seem to have an advantage in terms of oncologic outcomes by MDT [19]. A synergistic point of view could be found if it will be demonstrated that using upfront MDT followed or not by ADT does not reduce the castration-sensitive period, especially when ADT is administered after MDT failure.

In this single arm type of study, the TTCR was measured, which was quite impressive with median time of 48 months (assuming time zero is the last day of SBRT). Additionally, the median castration-sensitive phase after ADT administration was 29 months, suggesting that once ADT started, it took a median of 29 months before castration resistance developed. In the absence of randomized trials, a comparison could be conducted with previous historical publications according to which after first-line ADT, disease progression represents a common event due
Table 3 Statistical analytical results

\begin{tabular}{|c|c|c|c|c|}
\hline Covariate & ADT FS HR (95\% CI) & p-value & $\begin{array}{l}\text { ADT FS } \\
\text { (median, mo.) }\end{array}$ & p-value \\
\hline D’Amico risk group, n (\%) & & 0.342 & & 0.315 \\
\hline Very low/low & 1 & & 12.6 & \\
\hline Intermediate & $1.29(0.56-2.94)$ & & 13.8 & \\
\hline High/very high & $0.86(0.41-1.82)$ & & 18.2 & \\
\hline \multicolumn{5}{|l|}{ Gleason score sum } \\
\hline 6 & 1 & 0.15 & 9.7 & 0.15 \\
\hline 7 & $0.83(0.43-1.58)$ & & 18.5 & \\
\hline$>7$ & $0.55(0.27-1.1)$ & & 18.2 & \\
\hline \multicolumn{5}{|c|}{ PSA level at time of metastases, $\mathrm{ng} / \mathrm{ml}$} \\
\hline$<2 \mathrm{ng} / \mathrm{ml}$ & 1 & 0.069 & 18.2 & 0.065 \\
\hline$>2 \mathrm{ng} / \mathrm{ml}$ & $1.52(0.97-2.39)$ & & 15.6 & \\
\hline PSA doubling time & & 0.64 & & 0.917 \\
\hline$<5$ months & $1.12(0.69-1.81)$ & & 18.2 & \\
\hline$>5$ months & 1 & & 18.2 & \\
\hline Location of metastasis & & 0.64 & & 0.64 \\
\hline Node & 1 & & 18.2 & \\
\hline Bone & $1.15(0.63-2.1)$ & & 13.8 & \\
\hline Location of node metastasis & & 0.38 & & 0.38 \\
\hline N1 & 1 & & 18.2 & \\
\hline M1a & $0.79(0.47-1.34)$ & & 19.2 & \\
\hline \multicolumn{5}{|l|}{ No. of lesions } \\
\hline 1 & 1 & 0.74 & 18.2 & 0.74 \\
\hline$>1$ & $1.08(0.68-1.71)$ & & 18.2 & \\
\hline
\end{tabular}


to the development of the castration-resistant phase that generally occurs at a median follow-up of 24-36 months [2-4].

In this setting, it is worth mentioning the results of the STOMP [13] trial which compared MDT versus active surveillance in oligorecurrent PC. In the recent update of the study, authors reported 5-year CRPC-free survival rate of $76 \%$ versus $53 \%$ for MDT and surveillance arms, respectively. In our series, 5-year CRPC-free survival rate was about $65 \%$, which is reasonably in line with the current literature.

In contrast, the results of the TOAD trial [20] seem to be quite similar to ours in terms of ADTFS. This Australian paper compares delayed or immediate ADT administration for PSA-relapse PC patients and median time to ADT administration for the delayed arm is 18 months, which seems to be comparable to our median ADTFS. Yet, in our series, ADTFS was calculated from the last session of SBRT to the administration of ADT for the polymetastatic disease conversion. If we had to calculate ADTFS starting from the data of the biochemical relapse, our median ADTFS would reach 24 months, which is a reasonable achievement in this subgroup of patients. This scenario highlights the need of a standard definition of ADTFS in oligometastatic patients treated with MDT.

To the best of our knowledge, this is the first study that has demonstrated the synergistic action of sequential MDT and ADT to prolong the castration-sensitive "window". A possible explanation could be related to the different mechanisms of action by ablative irradiation and ADT. It is recognized that androgen-deprived PC metastasis could further metastasize and a clonal relationship among the different "waves" of PC metastases is reported. Thus, clonal heterogeneity inside each metastasis could be able to bypass ADT control by a selection process of sub-clones. This last phenomenon could lead toward a convergent path of therapeutic resistance. MDT "freezes" this process since the beginning thanks to its ablative effect, which does not depend from clonal ADT sensitivity.

The preliminary identification of the "real" oligorecurrent PC patients still remains therefore crucial before offering SBRT. In this scenario, the newer metabolic imaging remains crucial to overcome the so-called "iceberg theory" $[21,22]$. According to this last principle, MDT efficacy could be heavily affected by the limits in identifying the clinical disease diagnosed only using conventional imaging. The adoption of new metabolic tracers could optimize the selection process for MDT-strategy due to the possibility to detect macroscopic foci of disease at low PSA levels. In the present study both Choline and PSMA tracers were used to diagnose oligorecurrent disease, leading to potential biases. Indeed, in a previously published article from Mazzola and colleagues, PET-PSMA-guided MDT seems to offer an advantage in terms of ADT-free survival if compared to PET-choline-guided SBRT in the oligorecurrent PC [23].

The main interest of the current study for future research relies on its sample dimensions, follow-up duration, and its primary outcome (i.e., castration-resistant disease-free survival).

On the other hand, the possible limitations are (i) the retrospective nature of the study that may lead to some critical biases and (ii) different metabolic imaging used at time of oligorecurrence. Finally, last but not least, there was no comparison to another group of patients who did not get SBRT for oligorecurrent PC disease and managed with a more conventional/standard approach of observation and eventual ADT. For this reason, well-designed prospective trials are strongly advocated to validate the present retrospective study.

Funding Open access funding provided by Università degli Studi di Brescia within the CRUI-CARE Agreement.

Data availability Datasets are available upon reasonable request.

\section{Declarations}

Conflict of interest The authors have disclosed that they have not received any financial consideration from any person or organization to support the preparation, analysis, results, or discussion of this article. The authors declare that there is no conflict of interest.

Ethical approval All procedures performed in the study were in accordance with the ethical standards of the institutional and/or national research committee, and with the 1964 Helsinki declaration and its later amendments or comparable ethical standards.

Informed consent Specific and detailed consent to participate and for publication was obtained for each patients before the treatment. This work is published under the standard license to publish agreement.

Open Access This article is licensed under a Creative Commons Attribution 4.0 International License, which permits use, sharing, adaptation, distribution and reproduction in any medium or format, as long as you give appropriate credit to the original author(s) and the source, provide a link to the Creative Commons licence, and indicate if changes were made. The images or other third party material in this article are included in the article's Creative Commons licence, unless indicated otherwise in a credit line to the material. If material is not included in the article's Creative Commons licence and your intended use is not permitted by statutory regulation or exceeds the permitted use, you will need to obtain permission directly from the copyright holder. To view a copy of this licence, visit http://creativecommons.org/licenses/by/4.0/.

\section{References}

1. Cornford P, Bellmunt J, Bolla M, et al. EAU-ESTRO-SIOG guidelines on prostate cancer. Part II treatment of relapsing, metastatic, and castration-resistant prostate cancer. Eur Urol. 2017;71(4):630-42. 
2. Shore ND, Antonarakis ES, Cookson MS, et al. Optimizing the role of androgen deprivation therapy in advanced prostate cancer: Challenges beyond the guidelines. Prostate. 2020;80(6):527-44.

3. Harris WP, Mostaghel EA, Nelson PS, et al. Androgen deprivation therapy: progress in understanding mechanisms of resistance and optimizing androgen depletion. Nat Clin Pract Urol. 2009;6(2):76-85.

4. Pienta KJ, Bradley D. Mechanisms underlying the development of androgen independent prostate cancer. Clin Cancer Res. 2006;12(6):1665-71.

5. Sammon JD, Abdollah F, Reznor G, et al. Patterns of declining use and the adverse effect of primary androgen deprivation on all-cause mortality in elderly men with prostate cancer. Eur Urol. 2015;68:32-9.

6. Kroeze SGC, Henkenberens C, Schmidt-Hegemann NS, et al. Prostate-specific positron emission tomography-detected oligorecurrent prostate cancer treated with metastases-directed radiotherapy: of addition and duration of androgen deprivation. Eur Urol Focus. 2019;:S2405-4569(19):30270-6.

7. Triggiani L, Mazzola R, Magrini SM, et al. Metastasis-directed stereotactic radiotherapy for oligoprogressive castrationresistant prostate cancer: a multicenter study. World J Urol. 2019;37(12):2631-7.

8. Triggiani L, Alongi F, Buglione M, et al. Efficacy of stereotactic body radiotherapy in oligorecurrent and in oligoprogressive prostate cancer: new evidence from a multicentric study. Br J Cancer. 2017;116(12):1520-5.

9. Scher HI, Morris MJ, Stadler WM, et al. trial design and objectives for castration-resistant prostate cancer: updated recommendations from the prostate cancer clinical trials working group 3. J Clin Oncol. 2016;34(12):1402-18.

10. Wahl RL, Jacene H, Kasamon Y, et al. From RECIST to PERCIST: evolving considerations for PET response criteria in solid tumors. J Nucl Med. 2009;50:122S-150S.

11. https://ctep.cancer.gov/protocoldevelopment/electronic_applicatio ns/docs/CTCAE_v5_Quick_Reference_8.5x11.pdf

12. Palma DA, Olson R, Harrow $S$, et al. Stereotactic ablative radiotherapy versus standard of care palliative treatment in patients with oligometastatic cancers (SABR-COMET): a randomised, phase 2, open-label trial. Lancet. 2019;393(10185):2051-8.

13. Ost P, Reynders D, Decaestecker K, et al. Surveillance or metastasis-directed therapy for oligometastatic prostate cancer recurrence: a prospective, randomized, multicenter phase II trial. J Clin Oncol. 2018;36(5):446-53.
14. Phillips R, Shi WY, Deek M, et al. Outcomes of observation vs stereotactic ablative radiation for oligometastatic prostate cancer: the ORIOLE Phase 2 randomized clinical trial. JAMA Oncol. 2020;6(5):650-9.

15. Decaestecker K, De Meerleer G, Ameye F, et al. Surveillance or metastasis-directed Therapy for OligoMetastatic Prostate cancer recurrence (STOMP): study protocol for a randomized phase II trial. BMC Cancer. 2014;14:671.

16. Wibowo E, Wassersug RJ, Robinson JW, et al. How are patients with prostate cancer managing androgen deprivation therapy side effects? Clin Genitourin Cancer. 2019;17(6):480-1.

17. Nguyen C, Lairson DR, Swartz MD, et al. Risks of major long-term side effects associated with androgen-deprivation therapy in men with prostate cancer. Pharmacotherapy. 2018;38(10):999-1009.

18. Gillessen S, Omlin A, Attard G, et al. Management of patients with advanced prostate cancer: recommendations of the St gallen advanced prostate cancer consensus conference (APCCC) 2015. Ann Oncol. 2015;26(8):1589-604.

19. Connor MJ, Smith A, Miah S, et al. Targeting oligometastasis with stereotactic ablative radiation therapy or surgery in metastatic hormone-sensitive prostate cancer: a systematic review of prospective clinical trials. Eur Urol Oncol. 2020;3(5):582-93.

20. Duchesne GM, Woo HH, Bassett JK, et al. Timing of androgendeprivation therapy in patients with prostate cancer with a rising PSA (TROG 03.06 and VCOG PR 01-03 [TOAD]): a randomised, multicentre, non-blinded, phase 3 trial. Lancet Oncol. 2016;17(6):727-37. https://doi.org/10.1016/S1470-2045(16) 00107-8.

21. Mazzola R, Cuccia F, Figlia V, et al. New metabolic tracers for detectable PSA levels in the post-prostatectomy setting: is the era of melting glaciers upcoming? Transl Androl Urol. 2019;8(Suppl 5):S538-41.

22. Alongi F, Fersino S, Giaj Levra N, et al. Impact of 18F-choline $\mathrm{PET} / \mathrm{CT}$ in the decision-making strategy of treatment volumes in definitive prostate cancer volumetric modulated radiation therapy. Clin Nucl Med. 2015;40(11):e496-500.

23. Mazzola R, Francolini G, Triggiani L, et al. Metastasis-directed therapy (SBRT) guided by PET-CT (18)F-CHOLINE versus PETCT (68)Ga-PSMA in castration-sensitive oligorecurrent prostate cancer: a comparative analysis of effectiveness. Clin Genitourin Cancer. 2020;S1558-7673(20):30191-9.

Publisher's Note Springer Nature remains neutral with regard to jurisdictional claims in published maps and institutional affiliations. 